\title{
Analgesia e anestesia em gatos acometidos por fraturas e cirurgias ortopédicas: uma
}

\section{revisão}

\author{
Analgesia and anesthesia in cats affected by fractures and orthopedic surgery: a review \\ Analgesia y anestesia en gatos afectados por fracturas y cirugía ortopédica: una revisión
}

Ana Caroline Barros Correia do Monte

ORCID: https://orcid.org/0000-0002-4257-4753

Centro Universitário Maurício de Nassau, Brasil E-mail: anacarolinedomonte@ hotmail.com

\begin{abstract}
Resumo
Cirurgias ortopédicas para estabilização de fraturas diversas e luxações articulares estão entre os três procedimentos cirúrgicos mais comuns em gatos. A dificuldade em reconhecer a dor, à falta de conhecimento com relação ao uso adequado de analgésicos e o medo de efeitos colaterais dos fármacos podem levar a inadequada provisão de analgesia em gatos. O objetivo desta pesquisa foi descrever as principais ferramentas para avaliação da dor e para provisão de analgesia e anestesia em cirurgias ortopédicas de gatos. Esta pesquisa foi desenvolvida a partir da análise de publicações científicas sobre analgesia e anestesia em gatos, dando-se ênfase nas cirurgias ortopédicas. A escala multidimensional para avaliação de dor da UNESP-Botucatu é uma ferramenta poderosa e validada clinicamente para condição de pós operatória de cirurgias ortopédicas de gatos. O exame ortopédico pode fornecer informações significativas sobre a condição geral do paciente. As áreas de preocupação identificadas no exame ortopédico são posteriormente examinadas com radiografias ou tomografia computadorizada. A medicina veterinária conta com uma ampla variedade de fármacos analgésicos e anestésicos que podem e devem ser utilizados em felinos. Contudo, para provisão de analgesia adequada, é essencial o conhecimento sobre as particularidades metabólicas da espécie felina, respeitar as características dos fármacos analgésicos administrados durante o pré, trans e pós-cirúrgico, considerando, também, o impacto na escolha das doses e vias de administração. Um manejo analgésico adequado, através da avaliação regular clínica da dor, proporciona conforto e contribui com a recuperação e bem-estar do animal.
\end{abstract}

Palavras-chave: Luxações articulares; Avaliação da dor; Fármacos; Vias de administração.

\begin{abstract}
Orthopedic surgeries for stabilizing fractures and joint dislocations are among the three most common surgical procedures in cats. Difficulty in recognizing pain, lack of knowledge regarding the proper use of analgesics and fear of drug side effects can lead to inadequate provision of analgesia in cats. The aim of this research was to describe the main tools for pain assessment and for providing analgesia and anesthesia in orthopedic surgery in cats. This research was developed from the analysis of scientific publications on analgesia and anesthesia in cats, with emphasis on orthopedic surgeries. The UNESP-Botucatu multidimensional pain assessment scale is a powerful and clinically validated tool for post-operative condition of orthopedic surgery in cats. The orthopedic examination can provide significant information about the patient's general condition. Areas of concern identified on the orthopedic examination are further examined with radiographs or computed tomography. Veterinary medicine has a wide variety of analgesic and anesthetic drugs that can and should be used in felines. However, for the provision of adequate analgesia, knowledge about the metabolic particularities of the feline species is essential, respecting the characteristics of analgesic drugs administered during the pre, trans and post-surgery period, also considering the impact on the choice of doses and routes of management. Appropriate analgesic management, through regular clinical assessment of pain, provides comfort and contributes to the recovery and animal welfare.
\end{abstract}

Keywords: Joint dislocations; Pain assessment; Drugs; Routes of administration.

\section{Resumen}

Las cirugías ortopédicas para estabilizar diversas fracturas y dislocaciones articulares se encuentran entre los tres procedimientos quirúrgicos más comunes en gatos. La dificultad para reconocer el dolor, la falta de conocimiento sobre el uso adecuado de analgésicos y el miedo a los efectos secundarios de los medicamentos pueden provocar una administración inadecuada de analgesia en los gatos. El objetivo de esta investigación fue describir las principales herramientas para la evaluación del dolor y la provisión de analgesia y anestesia en la cirugía ortopédica en gatos. Esta investigación se desarrolló a partir del análisis de publicaciones científicas sobre analgesia y anestesia en gatos, con énfasis en cirugías ortopédicas. La escala de evaluación multidimensional del dolor UNESP-Botucatu es una herramienta poderosa y clínicamente validada para el estado postoperatorio de cirugía ortopédica en gatos. El examen 
ortopédico puede proporcionar información importante sobre el estado general del paciente. Las áreas de preocupación identificadas en el examen ortopédico se examinan más a fondo con radiografías o tomografía computarizada. La medicina veterinaria dispone de una amplia variedad de fármacos analgésicos y anestésicos que pueden y deben utilizarse en felinos. Sin embargo, para la provisión de una analgesia adecuada, es fundamental el conocimiento de las particularidades metabólicas de la especie felina, respetando las características de los fármacos analgésicos administrados durante el período pre, trans y posquirúrgico, considerando también el impacto en la elección de dosis y vías. de gestión. El manejo analgésico adecuado, mediante la evaluación clínica periódica del dolor, proporciona comodidad y contribuye a la recuperación y el bienestar del animal.

Palabras clave: Luxaciones articulares; Evaluación del dolor; Drogas; Rutas de administracion.

\section{Introdução}

Os gatos estão frequentemente sujeitos a agressões humanas, acidentes e doenças degenerativas que causam fraturas e luxações (Ramos et al. 2019; Ataide et al. 2020). Os principais sinais clínicos apresentados por esses pacientes são claudicação, impotência funcional, edema, hematoma e dor (Souza et al. 2019). A dor causa sofrimento, afeta a saúde e a qualidade de vida dos animais (Leece, 2020; Steagall, 2020). Portanto, caso a dor seja grave (acima de certo limiar), o animal deve ser anestesiado (Brondani et al., 2013).

A avaliação da dor baseia-se principalmente nas reações comportamentais, porém, a ampla variação de "personalidades" felinas e a variedade de comportamentos normais, tornam esta quantificação um desafio (Brondani et al., 2013; Belli, 2020). De fato, os gatos têm uma maneira única de expressar a dor. Diferentes indivíduos podem responder de forma completamente diferente a um estímulo nocivo (White, 2016). Portanto, a percepção e a avaliação da dor em gatos é uma tarefa complexa, que exige conhecimento e prática. Os principais sinais clínicos indicativos de dor em gatos e as ferramentas de avaliação da dor são apresentadas e discutidas nesta revisão.

A dor resultante de danos em tecidos moles é diferente da dor resultante de um dano ortopédico e, portanto, deve ser tratada com sua especificidade. Equivocadamente, os mesmos princípios do controle de dor aplicados inicialmente em caninos foram extrapolados para as espécies felinas. Isso provocou uma série de reações adversas e toxicidade, uma vez que o metabolismo dos fármacos nos gatos ocorre de forma limitada quando comparado aos caninos e outras espécies (Mathews et al., 2018).

A terapia medicamentosa em gatos deve considerar o metabolismo exclusivo desses animais, o que pode alterar tanto o perfil farmacocinético quanto o efeito farmacodinâmico do agente químico (Robertson, 2020). Os agentes analgésicos clássicos e mais comumente empregados em felinos, tais como os opióides, os anti-inflamatórios não esteroides (AINEs) e os anestésicos locais, são apresentados e discutidos com foco na anestesia em gatos submetidos a cirurgias ortopédicas (Mathews et al., 2018; Robertson, 2020).

Apesar dos avanços recentes nas técnicas de avaliação e controle da dor em gatos, as informações relacionadas especificamente a cirurgias ortopédicas nesta espécie são altamente fragmentadas. Portanto, o objetivo desta pesquisa foi descrever as principais ferramentas e técnicas para avaliação da dor e para provisão de analgesia e anestesia em cirurgias ortopédicas de gatos.

\section{Metodologia}

Esta pesquisa foi desenvolvida a partir da análise de publicações científicas sobre analgesia e anestesia em gatos, dando-se ênfase nas cirurgias ortopédicas. Os levantamentos foram realizados continuamente durante os meses de maio e junho de 2021. A pesquisa foi realizada no Google Scholar, PubMed, e Periódicos CAPES, que são bases de dados que oferecem acesso a textos completos e artigos publicados em revistas nacionais e internacionais. Os estágios de pesquisa e construção deste artigo incluíram (1) a formulação do problema; (2) definição dos termos de pesquisa; (3) coleta de 
dados/publicações; (4) avaliação de dados; (5) análise e interpretação; e (6) apresentação pública, tal como recomendado por Wee e Banister (2016).

A busca e seleção das fontes de informação deram-se por meio de cinco termos principais de pesquisa: "dor em gatos", "analgesia em gatos", "anestesia em gatos", "fraturas em gatos" e "cirurgias ortopédicas em gatos". Suas respectivas versões no inglês ("pain in cats", "analgesia in cats", "anesthesia in cats", "fractures in cats" and "orthopedic surgeries in cats") também foram utilizadas nos sistemas de busca de trabalhos. Foram selecionadas as referências consideradas mais pertinentes ao tema. O principal fator de exclusão foi o ano da publicação, excluindo-se trabalhos publicados anterior aos últimos 10 anos. O principal fator de inclusão foi o tema da pesquisa, incluindo-se, especificamente, trabalhos que contemplassem analgesia e anestesia em problemas ortopédicos em gatos.

\section{Resultados e Discussão}

Cirurgias ortopédicas para estabilização de fraturas diversas e luxações articulares estão entre os três procedimentos cirúrgicos mais comuns em gatos (Ataide et al. 2020). Há uma grande quantidade de trabalhos de pesquisa recentes sobre avaliação da dor em gatos (Belli, 2020; Moreira et al. 2021; Hernández-Avalos et al., 2021), mas poucos consideravam as especificidades da lesão ortopédica e o procedimento cirúrgico. Muitos trabalhos de pesquisa abordando a analgesia e anestesia durante procedimentos ortopédicos em gatos (Robertson, 2020), mas as informações sobre as técnicas de avaliação e controle da dor são altamente fragmentadas.

Os procedimentos cirúrgicos na medicina veterinária sofreram uma rápida especialização nas últimas décadas. Atualmente, os veterinários realizam rotineiramente substituições de articulações, neurocirurgias, reconstituição óssea e outros procedimentos complexos (Harrysson et al. 2015). Geralmente, os princípios básicos utilizados no tratamento de fraturas e luxações articulares em cães são aplicados em gatos (Mathews et al., 2018; Barbosa, 2019).

As diferenças clínicas, anatômicas e fisiológicas existentes entre gatos e cães demandam procedimentos específicos (Grimm et al., 2017; Mathews et al., 2018). Em geral, os ossos dos gatos são mais retos e delgados em relação aos ossos dos cães, os ângulos do ligamento patelar dos gatos são reduzidos em relação aos observados em cães (Arruda et al. 2018) e a capacidade dos felinos em metabolizar esses fármacos é limitada em relação a capacidade dos cães (Grimm et al., 2017).

As técnicas cirúrgicas para qualquer procedimento são adquiridas com a vivência. Para os cursos de medicina veterinária, é recomendado que os alunos iniciem seus treinamentos com métodos alternativos (como o uso de cadáveres) para depois operarem animais vivos (CONCEA, 2016).

\subsection{Identificação e avaliação da dor}

A lesão da fratura ou luxação resulta em uma resposta inflamatória local, na área envolvida ou sistemicamente. A presença da inflamação aumenta o grau de dor sentida após um procedimento cirúrgico, quando comparada com a de um procedimento de rotina (Mathews et al., 2018). Entretanto, comportamentos sugestivos de dor em gatos podem ser evidentes ou discretos, ou ainda ausentes, enquanto o animal sente dor (White, 2016). Isso dificulta a identificação, a avaliação e o controle da dor nessa espécie.

O exame ortopédico pode fornecer informações significativas sobre a condição geral do paciente e pode identificar potenciais lesões ósseas ou nervosas (Robertson, 2020). Se houver suspeita de dor nos ossos, pode-se empregar um toque suave durante o exame físico e ortopédico. Em muitos casos, devido à gravidade da dor e à progressão crônica, esses pacientes precisarão de manipulação mínima para demonstrar as áreas de dor. As áreas de preocupação identificadas no exame ortopédico são posteriormente examinadas com radiografias ou tomografia computadorizada (Mathews et al., 2018).

Geralmente, as mudanças comportamentais são os principais indicadores de dor em gatos. No entanto, 
comportamentos espontâneos indicativos de dor podem estar associados a outras emoções, como medo, apreensão ou ansiedade, e disfunção cognitiva (White, 2016). Alterações fisiológicos também podem ser influenciadas por outros fatores além da dor. Neste sentido, o conhecimento do comportamento normal do gato é extremamente importante ao avaliar a mudança no comportamento na presença potencial de dor (White, 2016; Leece, 2020).

Normalmente, após procedimentos ortopédicos, o movimento aumenta a intensidade da dor sentida, levando os gatos a se posicionarem em decúbito lateral. A interação identificará esta situação (Mathews et al., 2018). Para uma avaliação precisa do nível de dor, devemos considerar, simultaneamente, sinais comportamentais, parâmetros fisiológicos e avaliação qualitativa da resposta à interação e à palpação da área dolorosa (Brondani et al., 2013). Os principais sinais comportamentais e fisiológicos sugestivos de dor em gatos estão listados na Tabela 1.

Tabela 1. Sinais sugestivos de dor em gatos.

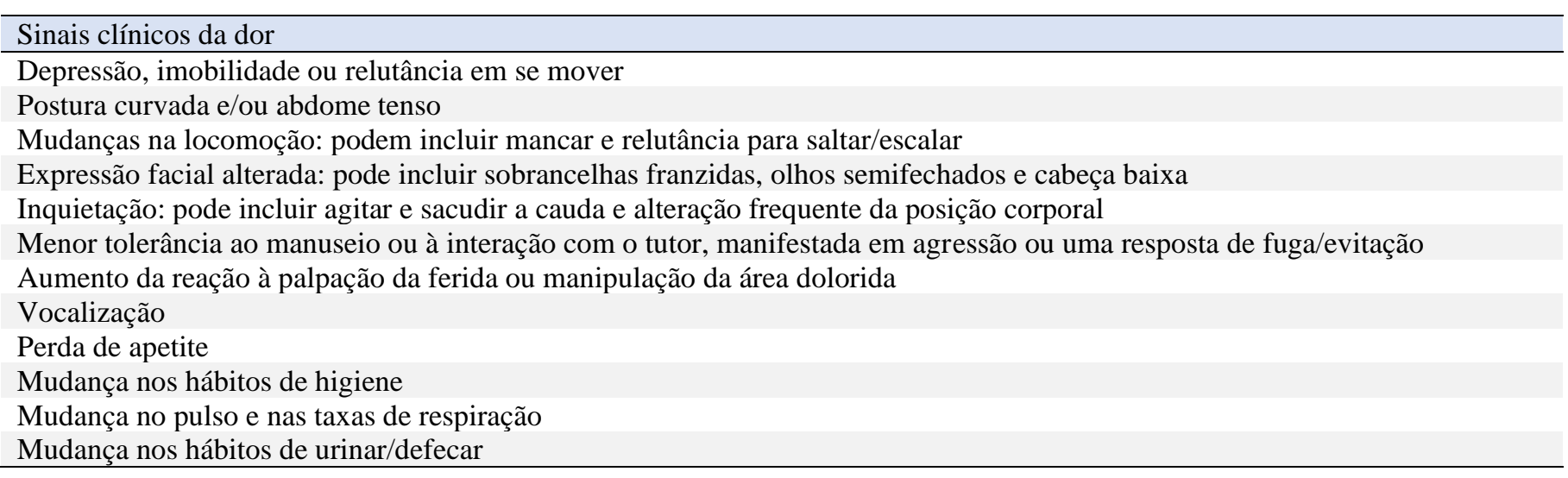
Fonte: Adaptado de White (2016).

Recentemente, a escala multidimensional para avaliação de dor da UNESP-Botucatu (EMAD U-B) foi validada clinicamente para condição de pós operatória de cirurgias ortopédicas de gatos (Belli, 2020). Trata-se de um instrumento validado, demonstrando validade, confiabilidade e sensibilidade, publicada em múltiplas línguas e aceita internacionalmente. Esta ferramenta é composta por um critério numérico estruturado a partir de 10 itens: postura, conforto, atividade, atitude, comportamentos diversos, reação à palpação da ferida cirúrgica, reação à palpação do abdômen / flanco, pressão arterial, apetite e vocalização. Cada item de escala é classificado numericamente $(0,1,2$ e 3), onde " 0 " indica nenhuma alteração ou estado normal e o valor mais alto indica a mudança mais marcada (ponto de corte para a analgesia de resgate). Além disso, contém diretrizes claras para seu uso, incluindo uma descrição detalhada dos comportamentos que devem ser observados em cada item de escala (Brondani et al., 2013).

\subsection{Especificidades do metabolismo de fármacos em felinos}

O organismo felino tem baixa capacidade para glicuronidação hepática, principalmente de medicamentos fenólicos, como o acetaminofeno e o propofol (Robertson, 2020). Neste contexto, estudos farmacocinéticos indicam que o paracetamol, propofol, carprofeno e ácido acetilsalicílico (aspirina) são eliminados de forma significativamente mais lenta em gatos do que em cães e humanos. Portanto, os fármacos excretados, como conjugados de glicuronídeos, podem ter meia vida prolongada e acumular-se no organismo dos felinos (Mathews et al., 2018).

Os gatos também são deficientes em várias outras enzimas de conjugação, incluindo citocromo P450. O citocromo P450 é uma importante superfamília de enzimas que metaboliza 70-90\% dos produtos farmacêuticos (Mathews et al., 2018). Nos gatos, o citocromo P-450 é praticamente inativo em virtude do polimorfismo. Além disso, diferenças na farmacocinética 
devido à menor capacidade de ligação às proteínas plasmáticas em gatos do que em cães podem resultar em quadros de intoxicação, uma vez que haverá maior concentração de fármaco livre, responsável por aumentar a sua disponibilidade (Robertson, 2020).

\subsection{Medicação Pré-Anestésica}

A medicação pré-anestésica (MPA) tem como possíveis finalidades: promover analgesia, sedação, redução da irritabilidade e agressividade, facilitando cateterismo intravenoso e indução de anestesia (Little, 2016). Existem várias opções de fármacos que podem ser utilizados na MPA em felinos, contudo, o emprego de opioides associados com benzodiazepínicos, acepromazina ou dexmedetomidina representa as combinações anestésica mais comuns nesta espécie.

A escolha do protocolo e doses utilizadas dependerá do estado físico, temperamento, história médica, quadro clínico do paciente e do procedimento planejado. Em alguns gatos indóceis, a combinação de fármacos dissociativos como cetamina ou tiletamina também pode ser considerada para obter contenção química (Mathews et al., 2018).

A administração de dexmedetomidina e opioide como MPA tem-se demonstrado eficaz em pacientes agressivos. Em gatos jovens e hígidos, a aplicação de midazolam sozinho ou em combinação com um opioide não é recomendada, uma vez que geralmente produzem excitação. Contudo, nos pacientes geriátricos e debilitados, esse mesmo fármaco é efetivo como sedativo, além de não causar efeitos cardiopulmonares (Grimm et al., 2017). A Tabela 2 contém os principais fármacos e suas respectivas doses de administração empregados na MPA de felinos.

Tabela 2. Principais fármacos e doses empregados na medicação pré-anestésica em gatos.

\begin{tabular}{lc}
\hline Fármaco & Dose $(\mathrm{mg} / \mathrm{kg})$ \\
\hline Acepromazina & $0,02-0,05$ \\
Diazepam & $0,1-0,5$ \\
Midazolam & $0,1-0,3$ \\
Xilazina & $0,5-2$ \\
Dexmedetomidina & $0,005-0,02$ \\
Morfina & $0,1-0,2$ \\
Metadona & $0,2-0,5$ \\
Meperidina & $2-10$ \\
Fentanil & $0,001-0,005$ \\
Tramadol & $1-2$ \\
Buprenorfina & $0,01-0,03$ \\
Butorfanol & $0,1-0,4$ \\
Cetamina & $2-5$ \\
Tiletamina Zolazepam & $2-5$ \\
\hline
\end{tabular}

Fonte: Little (2016); White (2017).

\subsection{Indutores Anestésicos}

Os fármacos indutores são geralmente anestésicos injetáveis, administrados por via intravenosa, produzem sedação e anestesia, e tem como finalidade induzir o paciente a um estado de inconsciência apropriado para intubação e manutenção anestésica. Características como hidrossolubilidade, estabilidade à luminosidade e temperatura, ação anestésica e analgésica, elevada margem de segurança, ausência de efeito cumulativo, com início rápido e relaxamento muscular e analgésico, são alguns dos requisitos necessários que o anestésico injetável deve possuir (Grimm et al., 2017).

$\mathrm{Na}$ rotina anestésica da espécie felina, os fármacos injetáveis frequentemente indicados para indução anestésica incluem os agentes dissociativos (cetamina ou tiletamina-zolazepam), tiopental, propofol, etomidato e alfaxalona (ainda indisponível comercialmente no Brasil) (Little, 2016). Na Tabela 3, estão listados os fármacos e as doses recomendadas para indução anestésica em felinos. 
Tabela 3. Fármacos e doses utilizados na indução intravenosos de felinos.

\begin{tabular}{lll}
\hline Fármaco & Sem MPA & Após MPA \\
\hline Cetamina* & $10 \mathrm{mg} / \mathrm{kg}$ & $5 \mathrm{mg} / \mathrm{kg}$ \\
Tiletamina-Zolazepam & $1 \mathrm{a} 3 \mathrm{mg} / \mathrm{kg}$ & $0,1-0,5 \mathrm{mg} / \mathrm{kg}$ \\
Propofol & $8 \mathrm{mg} / \mathrm{kg}$ & $6 \mathrm{mg} / \mathrm{kg}$ \\
Etomidato* & - & $2 \mathrm{mg} / \mathrm{kg}$ \\
Alfaxalona & $5 \mathrm{mg} / \mathrm{kg}$ & $2 \mathrm{a} 3 \mathrm{mg} / \mathrm{kg}$ \\
\hline
\end{tabular}

*Obrigatório o uso combinado com benzodiazepínico. Fonte: Little (2016).

Atualmente, o propofol é o fármaco hipnótico mais empregado na rotina, podendo ser associado a outros agentes analgésicos, como opióides de ultra-curta duração, cetamina e lidocaína. São poucas as informações relativas à farmacocinética do propofol na espécie felina, porém, atenção especial deve ser dada, visto que possuem diferenças importantes em relação à biotransformação hepática do propofol (Mathews et al., 2018).

\subsection{Manutenção}

\section{Anestesia Intravenosa Total (TIVA)}

A TIVA é uma técnica consagrada na anestesiologia humana (César et al. 2016), que tem sido preconizada também na prática anestésica em pequenos animais. Essa técnica utiliza, obrigatoriamente, agentes intravenosos, tanto para a indução como também para a manutenção anestésica, possibilitando o uso combinado de uma gama variável de diferentes agentes injetáveis, como os hipnóticos, analgésicos e bloqueadores neuromusculares. Na anestesiologia veterinária, destaca-se o emprego do propofol, derivados esteróides, como a alfaxalona, e anestésicos dissociativos (Mathews et al., 2018).

As principais vantagens da TIVA são: facilidade na administração, indução, manutenção e despertar suaves, não exposição aos anestésicos voláteis, ausência de poluição ambiental, estabilidade cardiorrespiratória, ausência de irritação das vias respiratórias, e mínimos efeitos colaterais. Como principais desvantagens estão: a necessidade de equipamento especial, custo relativamente elevado, ausência de mensuração em tempo real das concentrações plasmáticas e impossibilidade de reversão dos fármacos (César et al. 2016). A principal contraindicação para o emprego dessa técnica é seu uso em animais que possuam comprometimento hepático ou renal.

\section{Anestesia inalatória}

Os anestésicos gerais inalatórios são amplamente empregados em medicina veterinária, e apresentam vantagens farmacocinéticas expressivas sobre os anestésicos intravenosos. Com anestesia inalatória, é possível ter maior controle das concentrações dos fármacos no sangue e nos tecidos corporais, aumentando ou diminuindo o fluxo das substâncias que são inspiradas pelo paciente, o que permite mudanças rápidas na profundidade da anestesia e oferece um método simples para induzir, manter ou cessar a anestesia geral (Grimm et al., 2017; Pypendop, 2017).

Conduto, é necessário um aparelho específico para a administração dos agentes inalatórios, com fonte de oxigênio (O2), circuito respiratório para o paciente, absorvedor de dióxido de carbono (CO2) e um reservatório de gás compatível (Grimm et al., 2017). Os agentes anestésicos inalatórios são benéficos e seguros para a manutenção anestésica em felinos, pois são eliminados quase em sua totalidade pelo sistema respiratório (Little, 2016). O isoflurano é o principal anestésico inalatório em uso na anestesiologia veterinária, seguido pelo sevoflurano e, raramente, pelo desflurano e halotano (Little, 2016; Mathews et al., 2018). 


\subsection{Anestésicos locais}

Dentre todos os fármacos utilizados no manejo da dor, apenas os anestésicos locais conseguem abolir por completo a condução do impulso doloroso, causando analgesia completa, devido ao bloqueio sensorial e motor (Staffieri \& Steagall, 2017; Barletta \& Reed, 2019). Dentre as vantagens da utilização dessa técnica, destaca-se o melhor gerenciamento das vias aéreas, controle mais eficiente da dor pós-operatória, modificação da resposta fisiopatológica ao estresse cirúrgico e redução geral da mortalidade e morbidade (Mathews et al., 2018; Kraus, 2018).

Os anestésicos locais são bases fracas com um pKa (constante de dissociação) de 7,5 a 8,5 e são divididos em 2 categorias, com base em sua estrutura: aminoamidas ou aminoésteres. A estrutura geral de todos os agentes consiste em um grupo aromático conectado a uma amina terciária por meio de uma ligação amida ou uma ligação éster (Barletta \& Reed, 2019).

Dentre os representantes dos anestésicos locais, a lidocaína e a bupivacaína são os utilizados com maior frequência na medicina veterinária (Tabela 4). É importante mencionar que os felinos são mais propensos ao desenvolvimento de efeitos tóxicos quando empregados anestésicos locais do grupo amida, devido à deficiência hepática dos felinos para conjugar e metabolizar estes fármacos, caracterizando uma janela terapêutica relativamente estreita (White, 2017).

Tabela 4. Fármacos e doses de anestésicos locais utilizados na terapêutica da dor aguda em felinos.

\begin{tabular}{lcccc}
\hline \multicolumn{1}{c}{ Anestésico Local } & Início & Duração & Dose & Dose Toxica \\
\hline Lidocaína & $10-15 \mathrm{~min}$ & $60-120 \mathrm{~min}$ & $1-2,5 \mathrm{mg} / \mathrm{kg}$ & $10 \mathrm{mg} / \mathrm{kg}$ \\
Ropivacaína & $5-15 \mathrm{~min}$ & $180-300 \mathrm{~min}$ & $1,5 \mathrm{mg} / \mathrm{kg}$ & $2 \mathrm{mg} / \mathrm{kg}$ \\
Bupivacaína & $15-30 \mathrm{~min}$ & $240-360 \mathrm{~min}$ & $1 \mathrm{mg} / \mathrm{kg}$ & $2 \mathrm{mg} / \mathrm{kg}$ \\
\hline
\end{tabular}

Fonte: White (2017).

Além de promover a dessensibilização de uma área localizada do corpo, anestésicos locais contribuem com a redução da dependência de opioides e outras classes de fármacos utilizados na rotina anestésica. Além disso, os bloqueios regionais possibilitam a realização de procedimentos cirúrgicos com o paciente consciente, ou quando associados à anestesia geral, essa técnica promove redução significativa dos anestésicos gerais e analgesia pós-operatória, contribuindo com a estabilidade hemodinâmica do paciente (Barletta \& Reed, 2019).

\subsection{Anestesia Epidural}

Em alguns casos, é possível complementar ou até mesmo substituir a anestesia geral pela anestesia epidural, embora a combinação de ambas as técnicas seja mais comum, com o objetivo de reduzir as doses dos fármacos usados, além de diminuir a dor durante e após o procedimento cirúrgico (Little, 2016; Mathews et al., 2018; Barletta \& Reed, 2019). A anestesia peridural e os anestésicos intravenosos também podem ser utilizados sem a complementação da anestesia inalatória em procedimentos cirúrgicos caudais ao diafragma (Steagall, 2020).

Anatomicamente, a topografia do cone medular em felinos difere da espécie canina, uma vez que a medula espinhal nos gatos se afunila, formando o cone medular, estendendo-se deste a vertebra lombar L7 à segunda vértebra caudal Cd2. Por esse motivo, a punção no espaço sacrococcígeo é considerada como sitio mais seguro para realização da anestesia epidural em gatos domésticos, diminuindo os riscos de punção acidental e injeção intratecal de anestésicos (Otero et al. 2014), o que pode resultar em hipotensão severa, colapso cardiovascular e nos casos mais graves, a morte do paciente (Casoni et al. 2014).

De acordo com Steagall (2020), a administração epidural lombossacra de opioides, assim como os anestésicos locais, deve ser considerada para o tratamento da dor perioperatória felina. Além dos anestésicos locais, podem ser administrados por via epidural fármacos opioides, agonistas alfa2-adrenérgico, cetamina e AINE's, promovendo efeitos analgésicos com menos 
efeitos adversos que a administração sistêmica.

\subsection{Monitorização}

Durante o procedimento cirúrgico em gatos, assim como em outros animais, é necessário constante monitoramento dos parâmetros fisiológicos do paciente, tais como a pressão arterial, frequência respiratória e a temperatura (Uhlig et al., 2015; Grimm et al., 2017), objetivando, principalmente, assegurar o controle da profundidade anestésica (Grimm et al., 2017). Independentemente do procedimento anestésico, a vigilância associada ao monitoramento dos felinos durante a anestesia não deve ser negligenciada, contribuindo para a diminuição da morbidade e mortalidade relacionadas à anestesia nesta espécie.

Idealmente, a frequência cardíaca, a frequência respiratória, a pressão sanguínea e outras variáveis monitoradas devem ser registradas em intervalos regulares (5-10 min). Esses dados, em intervalos regulares, auxiliam na visualização das mudanças do estado do paciente durante o período anestésico. Em geral, um nível anestésico muito leve não consegue atingir todas as metas básicas da anestesia, enquanto uma anestesia muito profunda pode ter consequências cardiopulmonares adversas e levar o animal à morte (Grimm et al., 2017).

A administração adequada dos agentes anestésicos garante a inconsciência do paciente, amnésia, supressão da dor e movimento. Em contrapartida, os níveis excessivos de anestesia promovem hipoventilação e hipoxemia, redução do débito cardíaco, hipotensão, perfusão tecidual inadequada e recuperação prolongada (Grimm et al., 2017). Os sinais físicos indicativos da profundidade anestésica incluem a posição do globo, reflexo palpebral, tamanho das pupilas, resposta pupilar à luz, reflexo corneal, tônus do músculo ciliar e mandibular e resposta de movimento reflexo à nocicepção. (Uhlig et al., 2015)

\subsection{Controle da dor no pós-operatório}

De acordo com White (2017), para o controle da dor aguda em gatos, opta-se inicialmente, após triagem e avaliação clínica, pela utilização de fármacos opioides, devido ao seu bom efeito analgésico e poucos efeitos colaterais. Para controle da dor moderada a grave em gatos, a metadona tem se mostrado um fármaco seguro e eficaz, podendo ser aplicados pelas vias IV (lento) IM ou mesmo pela via transmucosa oral em pacientes mais indóceis e de difícil manipulação. Em casos de dor intensa, que necessitam de início rápido da ação, o fentanil IV ou a metadona podem ser administrados. Associado ao uso do opioide, se não houver contraindicação, deve-se considerar a administração de AINEs, promovendo efeito antiinflamatório e analgésico.

O uso de AINEs em gatos pode fornecer até 24 horas de analgesia, contudo, seu uso deve ser realizado com cautela. Há um potencial de toxicidade com o uso de AINEs em gatos, por causa da capacidade limitada dos felinos em metabolizar esses fármacos, resultando em duração prolongada com o potencial de acumulação (Grimm et al., 2017; Mathews et al., 2018).

\section{Considerações Finais}

Os gatos domésticos são frequentemente acometidos por fraturas e luxações articulares, que causam dor e, geralmente, precisam de procedimento cirúrgico. A dor deve ser identificada, quantificada subjetivamente e controlada por meio de anestésicos específicos. A identificação e quantificação da dor em felinos é uma tarefa complexa, principalmente devido a ampla variação comportamental da espécie, mas atualmente, a escala multidimensional para avaliação de dor da UNESP-Botucatu (EMAD U-B) é uma ferramenta poderosa e validada clinicamente para condição de pós operatória de cirurgias ortopédicas de gatos.

O exame ortopédico pode fornecer informações significativas sobre a condição geral do paciente e é provavelmente mais sensível do que outros testes de triagem para identificar potenciais lesões ósseas ou nervosas. As áreas de preocupação identificadas no exame ortopédico são posteriormente examinadas com radiografias ou tomografia computadorizada. 
A medicina veterinária conta com uma ampla variedade de fármacos analgésicos e anestésicos que podem e devem ser utilizados em felinos. Contudo, é essencial que o profissional tenha conhecimento sobre as particularidades dessa espécie, respeitando as características dos fármacos analgésicos administrados durante o pré, trans e pós-cirúrgico, levando também em consideração o impacto na escolha das doses, vias de administração, sobretudo as peculiaridades metabólicas exclusiva da espécie felina.

O regime analgésico deve ser adaptado individualmente à necessidade de cada paciente, assim, não existe uma receita pronta para o controle analgésico em gatos. Contudo, um manejo analgésico adequado durante o pré, trans e pós-cirúrgico, através da avaliação regular clínica da dor, proporciona conforto e contribui com a recuperação e bem-estar do animal.

Apesar dos avanços recentes, ainda são necessários mais estudos para ampliar o conhecimento e as técnicas especificas para avaliação e controle da dor durante procedimentos ortopédicas em gatos. As pesquisas devem avaliar o efeito de diferentes protocolos anestésicos (fármacos, doses, vias de administração) e associar suas respostas a fatores aleatórios, como raça, idade, grau do trauma e até mesmo o temperamento do animal.

\section{Referências}

Arruda, A. F. D., Muzzi, L. A., Lacreta Junior, A. C., Muzzi, R. A., Sampaio, G. R., Moreira, S. H., \& Mesquita, L. R. (2018). Radiographic assessment of the proximal tibial angles in dogs and cats with and without cranial cruciate ligament rupture. Pesquisa Veterinária Brasileira, 38(6), $1190-1195$.

Ataide, W. F., do Amaral, A. V. C., Bartoli, R. B. M., Bruno Filho, F. F., Romani, A. F., Regalin, D., \& de Souza Ramos, D. G. (2020). Estudo retrospectivo dos procedimentos cirúrgicos realizados em cães e gatos em um Hospital Veterinário Universitário na Região Centro-Oeste do Brasil. Brazilian Journal of Development, 6(6), 35413-35422.

Barbosa, L. M. (2019). Colocefalectomia em pequenos animais: estudo retrospectivo de 129 casos clínico-cirúrgicos. Trabalho de Conclusão de Curso II, do curso de graduação em Medicina Veterinária da Universidade Federal de Uberlândia. Uberlândia.

Barletta, M., \& Reed, R. (2019). Local anesthetics: pharmacology and special preparations. Veterinary Clinics: Small Animal Practice, 49(6), 1109-1125.

Belli, M. (2020). Validação clínica das escalas curta e longa da UNESP-Botucatu para avaliar a dor aguda em gatos. Dissertação (mestrado) - Universidade Estadual Paulista "Júlio de Mesquita Filho", Faculdade de Medicina de Botucatu.

Brondani, J. T., Mama, K. R., Luna, S. P., Wright, B. D., Niyom, S., Ambrosio, J., \& Padovani, C. R. (2013). Validation of the English version of the UNESPBotucatu multidimensional composite pain scale for assessing postoperative pain in cats. BMC Veterinary Research, 9(1), 1-15.

Casoni, D., Rohrbach, H., \& Spadavecchia, C. (2014). Total spinal anaesthesia following spinal lumbosacral injection of bupivacaine 0.5 per cent in a cat. Veterinary Record Case Reports, 2(1).

César, T. Z., César, T. Z., Combat, A. R., \& de Leão, B. C. C. (2016). Anestesia venosa: análise do desempenho quando comparada à anestesia com anestésicos inalatórios. Rev. Med. Minas Gerais, 26(supl 27), 4-10.

CONCEA. (2016). Conselho Nacional de Controle de Experimentação Animal. Orientação Técnica CONCEA no 9 de 18 de agosto de 2016. https://www.in.gov.br/materia/-/asset_publisher/Kujrw0TZC2Mb/content/id/21791247/DiarioOficialdaUniao

Grimm, K., Lamont, L., Tranquilli, W., Greene, S., \& Robertson, S. (2017). Lumb \& Jones Anestesiologia e analgesia veterinária. Editora Roca.

Harrysson, O. L., Marcellin-Little, D. J., \& Horn, T. J. (2015). Applications of metal additive manufacturing in veterinary orthopedic surgery. Jom, 67(3), 647654.

Hernández-Avalos, I., Flores-Gasca, E., Mota-Rojas, D., Casas-Alvarado, A., Miranda-Cortés, A. E., \& Domínguez-Oliva, A. (2021). Neurobiology of anesthetic-surgical stress and induced behavioral changes in dogs and cats: A review. Veterinary world, 14(2), 393.

Kraus, B. L. H. (2018). Anesthetic considerations for orthopedic surgical patients. In Small Animal Anesthesia and Pain Management (pp. 265-278). CRC Press.

Leece, E. (2020). Feline: what's new in pain recognition and scoring?. In BSAVA Congress Proceedings 2020, BSAVA Library, $155-155$.

Little, S. E. (2016). O gato: medicina interna. Rio de Janeiro: Roca, 978-989.

Mathews, K. A., Sinclair, M., Steele, A. M., \& Grubb, T. (Eds.). (2018). Analgesia and anesthesia for the ill or injured dog and cat. Wiley Blackwell.

Moreira, C. M., Oliveira, R. L., Costa, G. A., Corgozinho, K. B., Luna, S. P., \& Souza, H. J. (2021). Evaluation of tumescent local anesthesia in cats undergoing unilateral mastectomy. Veterinary Anaesthesia and Analgesia, 48(1), 134-141.

Otero, P. E., Zaccagnini, A. S., Fuensalida, S. E., Verdier, N., Sclocco, M., \& Portela, D. A. (2014). Use of electrical nerve stimulation to monitor lumbosacral epidural needle placement in cats. Veterinary anaesthesia and analgesia, 41(3), 325-329. 
Research, Society and Development, v. 10, n. 12, e367101220524, 2021

(CC BY 4.0) | ISSN 2525-3409 | DOI: http://dx.doi.org/10.33448/rsd-v10i12.20524

Pypendop, B. (2017). Inhalation and Balanced Anesthesia. Feline Anesthesia and Pain Management, 89-104.

Ramos, D., Reche-Junior, A., Hirai, Y., \& Mills, D. S. (2020). Feline behaviour problems in Brazil: a review of 155 referral cases. Veterinary Record, 186(16), e9-e9.

Robertson, S. (2020). Anesthetic protocols for dogs and cats. High-Quality, High-Volume Spay and Neuter and Other Shelter Surgeries, 153-192.

Souza, M. D. J. D., Ferreira, M. P., Amadori, A., Carneiro, R. K., Junqueira, A. M. C., Hergemöller, F., \& Sebastião, G. A. (2019). Osteossíntese com placa e pino em cães e gatos. Acta scientiae veterinariae. 47(1), 474.

Staffieri, F., \& Steagall, P. (2017). Local Anesthetics and Loco-regional Techniques. Feline Anesthesia and Pain Management, 67-88.

Steagall, P. V. (2020). Analgesia: What Makes Cats Different/Challenging and What Is Critical for Cats? Veterinary Clinics: Small Animal Practice, 50(4), 749-767.

Steagall, P. V., Monteiro, B. P., Lavoie, A. M., Frank, D., Troncy, E., Luna, S. P., \& Brondani, J. T. (2013). Validation of the French version of the UNESPBotucatu multidimensional composite pain scale for assessing postoperative pain in cats. Canadian Veterinary Journal, 58(1), 56-64.

Uhlig, C., Krause, H., Koch, T., de Abreu, M. G., \& Spieth, P. M. (2015). Anesthesia and monitoring in small laboratory mammals used in anesthesiology, respiratory and critical care research: a systematic review on the current reporting in top-10 impact factor ranked journals. PLoS One, 10(8), e0134205.

Wee, B. V., \& Banister, D. (2016). How to write a literature review paper? Transport Reviews, 36(2), 278-288.

White, K. L. (2016). Assessment of acute pain in cats: signs, tools and limitations. Companion Animal, 21(8), 458-465.

White, K. L. (2017). Treatment of acute pain in cats. Companion Animal, 22(1), 8-14. 